\section{New charity dental unit on the road}

More homeless and vulnerable people across the UK will be able to access free dental care after Dentaid launches its second charity dental unit at the BDIA Dental Showcase.

The vehicle will visit homeless shelters, soup kitchens and day centres with teams of volunteers providing dental screening, emergency treatment and oral health advice for people who find it difficult to access dental care.

The new unit has been supported by Bupa Dental Care, which has chosen Dentaid as its official charity partner. Bupa dental practices will be supporting Dentaid's UK work with a variety of fundraising initiatives involving patients and employees.

Since Dentaid launched its first mobile dental unit in 2016, the charity has been travelling the country providing free treatment for many of the most vulnerable people in our society. Homeless people, children in areas of poverty, fishermen and those living in places where it is difficult to register with an NHS practice, have all benefitted from clinics carried out in schools, community buildings, hostels and fishing ports from Cornwall to Aberdeenshire.

In the first nine months of 2019, 475 homeless and hard-to-reach people have been seen in Dentaid clinics and the charity receives

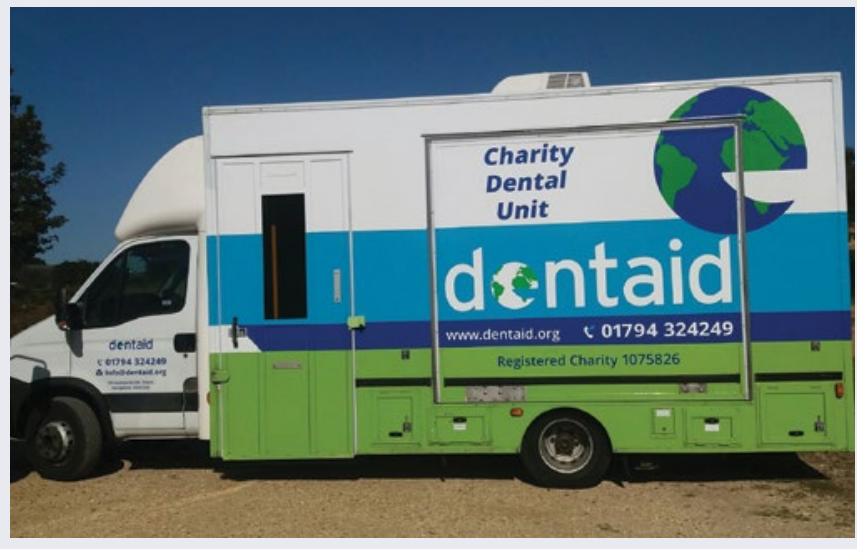

requests for new projects every week. The new unit will enable Dentaid to run more clinics with one vehicle stationed in the south of England and one in the north.

In addition to supporting the new dental unit, Bupa Dental Care has also provided funding, via its apprenticeship levy, for Dentaid to hire an administration apprentice and made Dentaid the beneficiary of its Pennies scheme - a digital charity box available in all Bupa Dental Care practices.

For more information about Dentaid visit www.dentaid.org.

\title{
All patients can benefit
}

Wisdom Toothbrushes offers a range of highly effective oral healthcare products to help all your patients improve their oral hygiene.

Leading the pack is the Wisdom Clean Between Interdental Brushes - the UK's No.1 selling rubber interdental brushes. Now with a new stronger stem, they feature a flexible, tapered head and super-soft micro-fine rubber filaments that slide smoothly between the teeth, massaging and stimulating the gingiva for a thorough yet gentle clean. Clinically proven to reduce gum disease, they are suitable for use around implants, bridges, crowns and orthodontic appliances and are an ideal solution for patients who find wire interdental brushes difficult or uncomfortable to use.

Also in the portfolio are the Wisdom Pro-flex Wire Interdental Brushes, featuring an ergonomic handle and curved profile that allow access to even hard the reach areas of the mouth.

Discover these and more solutions from Wisdom

Toothbrushes today! Visit www.wisdomtoothbrushes.com or call 01440714800 .

\section{Your helping hand}

Litigation is a very real threat in modern dentistry.

As such, you need peace of mind that you have professionals on your side who both understand the dental industry and whom have years of experience in dealing with cases within the sector.

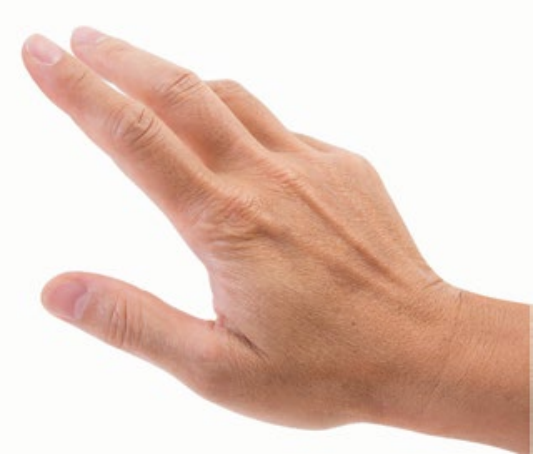

The team at

lawyers4dentists provide only the most professional legal advice and services from specialist dental experts.

Working closely with you and your practice, the team will go to every length to ensure that you have all of the information, advice and support you need in order to be best represented going forward.

To find out more, call the team at lawyers4dentists today. Call 0845 3455060 or 0754 DENTIST. Email info@4dentistsgroup.com or visit www.4dentistsgroup.com.

\section{Meet the demand}

With increasingly high expectations, today's patients want to be confident that you can offer a dental implant that not only functions like a natural tooth, but looks like one too. That's why many clinicians choose the $\mathrm{Z} 1$ implant system manufactured by TBR.

The $\mathrm{Z} 1$ remains the world's only tissue-level implant to combine a titanium body with a unique zirconia collar in one seamless component. This cutting-edge design offers excellent hard and soft tissue integrative properties, which enable clinicians to achieve an optimal, natural-looking result.

Boasting an impressive $98.6 \%$ success rate, it's no wonder that the $\mathrm{Z} 1$ implant is the first-choice solution for many practitioners.

For more information on the $\mathrm{Z} 1$ implant, visit tbr.dental, email support@denkauk.com or call 08007076212. 